\title{
Local density of states, spectrum, and far-field interference of surface plasmon polaritons probed by cathodoluminescence
}

\author{
M. Kuttge, ${ }^{1}$ E. J. R. Vesseur, ${ }^{1}$ A. F. Koenderink, ${ }^{1}$ H. J. Lezec, ${ }^{2}$ H. A. Atwater, ${ }^{2}$ F. J. García de Abajo, ${ }^{3}$ and A. Polman ${ }^{1}$ \\ ${ }^{1}$ Center for Nanophotonics, FOM-Institute of Atomic and Molecular Physics, Sciencepark 113, 1098 XG Amsterdam, \\ The Netherlands \\ ${ }^{2}$ Department of Applied Physics California Institute of Technology, Pasadena, California 91125, USA \\ ${ }^{3}$ Instituto de Óptica, CSIC, Serrano 121, 28006 Madrid, Spain \\ (Received 15 October 2008; published 17 March 2009)
}

\begin{abstract}
The surface plasmon polariton (SPP) field intensity in the vicinity of gratings patterned in an otherwise planar gold surface is spatially resolved using cathodoluminescence (CL). A detailed theoretical analysis is presented that successfully explains the measured CL signal based upon interference of transition radiation directly generated by electron impact and SPPs launched by the electron and outcoupled by the grating. The measured spectral dependence of the SPP yield per incoming electron is in excellent agreement with rigorous electromagnetic calculations. The CL emission is shown to be similar to that of a dipole oriented perpendicular to the surface and situated at the point of electron impact, which allows us to establish a solid connection between the CL signal and the photonic local density of states associated to the SPPs.
\end{abstract}

DOI: 10.1103/PhysRevB.79.113405

Surface plasmon polaritons (SPPs) are electromagnetic waves bound to the interface between a metal and a dielectric. ${ }^{1}$ The strong coupling between optical radiation and the collective plasmon oscillations of the conduction electrons near the metal surface leads to complex SPP behavior that can give rise to large field enhancements, ${ }^{2}$ negative refraction, ${ }^{3}$ and many other interesting phenomena resulting from sub-100 nm optics intrinsic to SPPs at visible and nearinfrared frequencies.

A major bottleneck in nearly all studies on the fundamental properties of SPPs is the limited spatial resolution by which plasmonic phenomena can be measured. Optical microscopy suffers from the diffraction limit, whereas nearfield microscopy has a resolution limited by the tip aperture to typically $100 \mathrm{~nm}$. In contrast, SPPs can also be excited using high-energy electron irradiation, with the electron beam focused to a nanometer size spot, thus enabling the excitation of SPPs with nanoscale resolution. Only a few studies of electron-beam irradiation of plasmonic structures have been reported, mainly focusing on measurements of the mode distribution of plasmons in nanoparticles ${ }^{4-6}$ or plasmon losses in planar surfaces. ${ }^{7,8}$ However, no detailed analysis of the different emission components and their interaction has been presented and no connection of the emission to the plasmonic density of states has been established.

In this Brief Report, we use electron-beam irradiation to study fundamental properties of SPPs propagating on a twodimensional substrate. In particular, we use the electron beam of a scanning electron microscope (SEM) impinging on a single-crystalline Au substrate as a nanoscale source of SPPs with a broad spectral range. Our key findings are as follows. (1) We have developed a model of cathodoluminescence (CL) emission which includes the excitation of SPPs, eventually outcoupled from the $\mathrm{Au}$ surface, and transition radiation (TR), ${ }^{9,10}$ as well as the interference of the two components; (2) extensive CL measurements performed over the visible spectrum and at distances up to a few microns from the grating are well reproduced by this model; (3) we measure the SPP generation yield per electron and find it to be in
PACS number(s): 73.20.Mf, 41.60.- $-\mathrm{m}, 78.60 . \mathrm{Hk}$

excellent agreement with rigorous electromagnetic calculations of this quantity; (4) the CL emission is found to be similar to that of a dipole positioned at the position of electron impact; and (5) this similarity allows us to establish a solid connection between the CL signal and the photonic local density of states (LDOS) associated to SPPs.

In our experiments, we use spatially resolved cathodoluminescence spectroscopy, a technique that combines scanning electron microscopy with the detection of optical radiation which is emitted from the sample. In view of the complexity of the CL process, we first review its basic features. In a qualitative picture, an impinging electron produces a localized perturbation of the density of conduction electrons in the region where it impinges onto the metal. This perturbation together with the incoming electron charge behaves effectively as a dipole oriented normal to the surface (see below) that serves as a source of SPPs. ${ }^{11}$ The SPPs propagate until they interact with a grating structured into the surface, where they are decoupled from the surface as light that can be detected in the far field [see schematic Fig. 1(a)]. The impinging electron also produces TR (Refs. 9 and 10), the angular dependence of which is shown in Fig. 1(a). The angular emission pattern for TR resembles very much that of a classical dipole placed infinitesimally close above a planar metal surface also shown in Fig. 1(a). The SPP and TR components of the emission are mutually coherent, so they produce interference in the far field where the CL detection takes place. To illustrate the interference, the colored background of Fig. 1(a) shows the two-dimensional near-field intensity distribution calculated using a two-dimensional boundary element method ${ }^{12}$ for an electron incident on a $\mathrm{Au}$ surface. The induced electric field is calculated for a geometry that is translationally invariant along the direction perpendicular to the paper and for wave vector components only in the plane of the paper. The separate emission components from TR and outcoupled SPPs as well as their interference are easily identified.

In our experiments, the sample consists of a single-crystal Au pellet of $1 \mathrm{~mm}$ thickness of which the surface was chemi- 

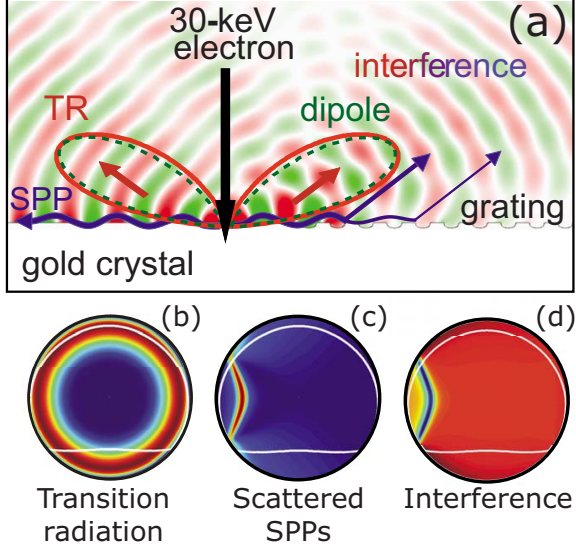

FIG. 1. (Color online) (a) Schematic of an electron impinging on a gold surface generating SPPs and TR (all calculations are done for a wavelength of $700 \mathrm{~nm}$ and the electron incident $1000 \mathrm{~nm}$ away from the grating). The colored background shows the twodimensional interference pattern of SPPs outcoupled by a grating with $400 \mathrm{~nm}$ period and TR calculated using the boundary element method. The calculated angular dependence of the emitted TR intensity is shown as a polar plot curve in red; the angular dependence of the emission of a dipole placed close to the metal is shown in dashed green. (b) Azimuthal projection of calculated TR, (c) radiation from scattered SPPs, and (d) interference of TR and SPPs in the far field. All plots are normalized to their maximum value. The white contours reflect the collection range of the mirror. The grating is orientated vertically and its edge is located at the origin of the plots.

cally polished down to nanometer roughness. Linear grating structures were milled into the surface with a $30 \mathrm{keV} \mathrm{Ga}^{+}$ focused ion beam. Gratings consisting of ten grooves were carved with periods $a=300,400$, and $500 \mathrm{~nm}$, respectively, and groove width $\approx a / 2$, depth $\approx 50 \mathrm{~nm}$, and length $50 \mu \mathrm{m}$. Spatially resolved CL spectroscopy was performed in a SEM using a $30 \mathrm{keV}$ electron beam from a field-emission source focused onto the sample to a $\sim 5 \mathrm{~nm}$ diameter spot. The actual resolution of this technique is slightly larger than the beam spot, with an upper limit dictated by the extension of the evanescent electric field of the electron $\sim v /(2 \omega)$, where $v$ is the electron velocity and $\omega$ is the light frequency [e.g., $\sim v /(2 \omega)=16 \mathrm{~nm}$ for $30 \mathrm{keV}$ electrons at a wavelength of $600 \mathrm{~nm}$ ]. A parabolic mirror positioned above the sample collects light emitted above it. Light is then spectrally resolved using a charge coupled device (CCD) array detector (bandwidth $\approx 10 \mathrm{~nm}$ ) after passing a monochromator. The mirror acceptance solid angle is $1.4 \pi$. Spectra were corrected for system response, which was determined by normalizing measured raw data from a planar Au sample (no grating) to the calculated TR spectrum for Au. ${ }^{10}$ This normalization allows us to compare the measured data to calculations on absolute scales and to compensate for the spectral response of our measurement system.

Figures 2(a) and 2(c) show the measured CL intensity plotted as a function of wavelength and distance to the grating for grating periods of $a=300 \mathrm{~nm}$ and $a=400 \mathrm{~nm}$, respectively. Several characteristic features are clearly resolved in these plots:

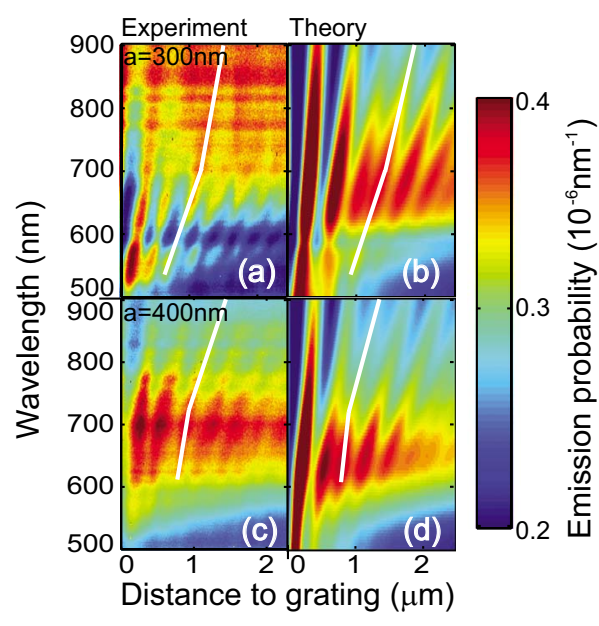

FIG. 2. (Color online) Cathodoluminescence intensity for 30 $\mathrm{keV}$ electrons incident on single-crystalline Au plotted as a function of detection wavelength and distance to the grating of Fig. 1. The grating period is $300 \mathrm{~nm}$ in (a) and (b) and $400 \mathrm{~nm}$ in (c) and (d). (a) and (c) are measurements for gratings carved in a single-crystal gold sample. (b) and (d) are calculations. The experimental data were corrected for system response.

(i) For wavelengths below $600 \mathrm{~nm}$, the CL intensity decays with distance from the grating. This is attributed to the strong damping of SPPs due to ohmic losses in this wavelength range close to the SPP resonance at $540 \mathrm{~nm}$. The observed CL decay with distance corresponds well to the decay length calculated using the dielectric constant for $\mathrm{Au}$. At longer wavelengths, the SPP propagation distance is calculated to be larger than the scan range in Fig. 2 and only a weak decay is observed. ${ }^{7,8}$ These data confirm that a large portion of the CL intensity in Fig. 2 is due to outscattered SPPs.

(ii) Superimposed on the decay, we observe a periodic modulation of the CL intensity with distance with a period of about half the detection wavelength. This is clear evidence of the noted interference between TR and SPPs contributions to the emission, which can be either constructive or destructive depending on the phase difference $\phi$ [see Eq. (1)], increasing linearly with $d$.

(iii) For the longer wavelengths the amplitude of the oscillations decreases with distance roughly as $1 / \sqrt{d}$, which is consistent with the distance dependence exhibited by any planar surface excitation originating in a point source and, in particular, the SPP waves launched at the position of the electron beam. ${ }^{13}$

To model our experiment, we have calculated the emission probability into SPPs and TR for an electron incident on a gold surface. Figures 1(b)-1(d) show the calculated angleresolved maps of the light intensity distribution due to TR (b), outcoupled SPPs (c), and their interference (d), respectively, all calculated for $30 \mathrm{keV}$ electrons on $\mathrm{Au}$ at a wavelength of $600 \mathrm{~nm}$. The TR is clearly isotropic in the azimuthal plane, while the scattered SPP distribution is highly anisotropic due to the presence of the grating. The solid angle of the parabolic mirror used to collect the CL is indicated by the white contours in Figs. 1(b)-1(d).

The experimentally collected CL intensity corresponds to 
the integral over the emission angles $\Omega$ of the interference within the collection solid angles of the CL mirror,

$$
I_{\mathrm{CL}}=\int_{\text {mirror }} d \Omega\left|A_{\mathrm{SPP}} \mathbf{S}(\Omega) e^{i \phi}+\mathbf{f}_{\mathrm{TR}}(\Omega)\right|^{2},
$$

where $A_{\mathrm{SPP}}$ is the SPP-excitation amplitude, $\mathbf{S}$ is the normalized in-plane far-field amplitude of a SPP scattered by the grating, $\mathbf{f}_{\mathrm{TR}}$ is the far-field amplitude of TR, and $\phi$ is the phase difference between the SPP and TR emission components. This expression is exact at large distances $d$ between the beam spot and the grating and, in particular, the TR field decays as $1 / d$ at the position of the grating, whereas the SPP field becomes dominant near the grating, since it dies off only as $1 / \sqrt{d}$, so that TR scattering by the grating is negligible for $d$ above a few hundred nanometers. In our calculations, we have used rigorous analytical solutions of Maxwell's equations for $A_{\mathrm{SPP}}$ (Ref. 14) and $\mathbf{f}_{\mathrm{TR}} \cdot{ }^{15}$ In particular, $A_{\mathrm{SPP}}$ is obtained from the plasmon-pole contribution to the field produced by the electron crossing a semi-infinite metalvacuum boundary. The dependence of $I_{\mathrm{CL}}$ on the separation $d$ between electron beam and grating comes exclusively through the relative phase of SPP and TR contributions $\phi$ $\propto d$. The grating scattering factor is approximated as $\mathbf{S}(\Omega)$ $=S_{0} /\left(k_{x}-2 \pi / a-i \Gamma / 2\right)$, where $k_{x}$ is the projection of the emitted photon momentum along the surface direction perpendicular to the grating, $a$ is the grating period, and $\Gamma$ $=1 /(\mathrm{Na})$ accounts for inelastic and radiative damping in the grating. We assume a mean-free path of $N=5$ periods and a typical scattering efficiency $S_{0}=40 \% .{ }^{16}$ These two constants are the only adjustable parameters in our model.

The calculated CL emission for two gratings of periods of 300 and $400 \mathrm{~nm}$ is shown in Figs. 2(b) and 2(d). We observe overall good agreement between measurements and calculated CL signal. Both the overall intensity as well as the periodic oscillations are very well resolved. Additionally, the period of the oscillations is a nonlinear function of wavelength and depends on the pitch of the grating. This becomes clear by comparing the data with the white lines in Fig. 2, which are intended to guide the eyes through a range of interference maxima. These curves both show a kink near a wavelength of $700 \mathrm{~nm}$ and quite different slopes above 700 $\mathrm{nm}$, both in experiment and theory. Indeed, for the $400 \mathrm{~nm}$ grating the first-order grating coupling mode for SPPs with a wavelength longer than $700 \mathrm{~nm}$ lies outside the acceptance range of the mirror. This provides further evidence for coherent interaction between SPPs scattered from the grating and TR. From the good agreement of experiment and theory, we conclude that far-field-emission measurements are well described by the model of Eq. (1).

In a next step, we have extracted from the spatially resolved CL data in Fig. 2 the spectral distribution of SPPs generated by the electron beam. At each wavelength, the CL intensity was integrated over the distance range of Fig. 2 correcting for the (measured) decay (thereby averaging out the oscillations). The obtained spectrum was then corrected for TR acquired at a position far away from the grating. Figure 3(a) shows the result of this analysis for three different values of the grating pitch (symbols). A quite similar

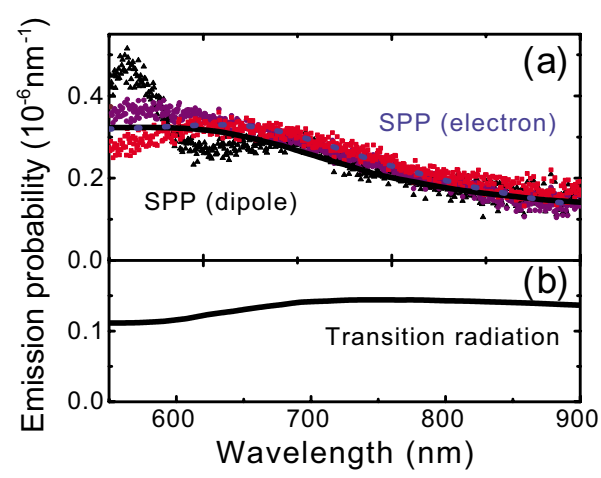

FIG. 3. (Color online) (a) Spectrum of surface plasmon polaritons on single-crystal gold excited by $30 \mathrm{keV}$ electrons and integrated over the measurement range of Fig. 2 next to gratings with three different grating pitches $[a=300 \mathrm{~nm}$-black triangles, 400 $\mathrm{nm}$-violet dots, and $500 \mathrm{~nm}$-red squares]. The solid lines show calculated spectra for SPPs generated by a $30 \mathrm{keV}$ electron impinging on the surface (blue: dashed) and by a dipole positioned at the surface (black: solid). (b) Calculated transition radiation spectrum for $30 \mathrm{keV}$ electrons on $\mathrm{Au}$.

spectral shape is observed for all gratings consistent with the fact that the generated SPP spectrum is independent of grating pitch. Figure 3(a) also shows the calculated SPP spectrum under $30 \mathrm{keV}$ electron excitation as well as the spectrum of SPPs that would be excited by a point dipole positioned at the metal surface. The dipole strength is taken as frequency independent in our calculations. These two calculations are rigorous analytical solutions of Maxwell's equations. ${ }^{14}$ The SPP emission rate of the dipole is plotted by normalizing the spectral integral of TR and the far-field dipole radiation. The small difference between these two calculated SPP spectra is due to the fact that the electron excites SPPs as it travels along its path, while the modeled point dipole is stationary. For comparison, Fig. 3(b) shows the calculated transition radiation spectrum which amounts to $30 \%$ of the SPP signal. The calculated spectra in Fig. 3(a) agree well with the experimentally determined spectra for longer wavelengths. The variations in the measured spectra for shorter wavelengths are ascribed to differences in coupling characteristics of the gratings and uncertainties in the correction for the SPP decay close to the SPP resonance.

The oscillations with distance in Fig. 2 are reminiscent of experiments performed by Drexhage, who studied the spontaneous emission of a rare-earth complex in front of a metal mirror ${ }^{17}$ It is well known that the spontaneous emission rate of optical emitters is proportional to the LDOS, as first experimentally demonstrated by Drexhage. Now, this allows us to construct an intuitive picture of the radiation generated by an impinging electron, which was shown to have an angular TR emission distribution (Fig. 1) and spectral coupling to SPPs similar to that of a point dipole [Fig. 3(a)]. The decay of this effective dipole into SPPs and TR is governed by the LDOS. The total decay rate is related to the LDOS $\rho$ through the dipole decay rate $\Gamma=\left(4 \pi^{2} \omega D^{2} / \hbar\right) \rho,{ }^{18}$ where $\omega$ is the emission frequency and $D$ is the dipole strength. The LDOS can be obtained from the projected field induced by the dipole on itself $E_{\perp}^{\text {ind }}$ as ${ }^{19}$ 


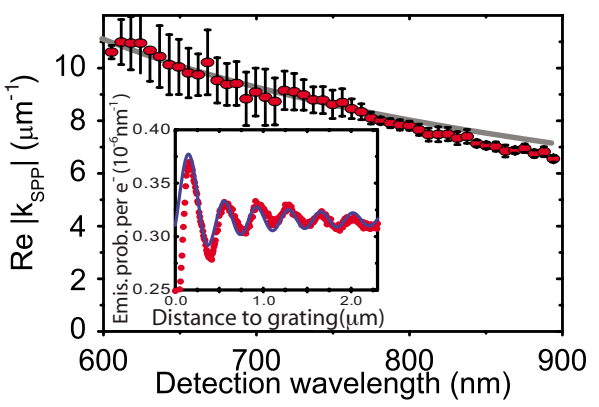

FIG. 4. (Color online) The main figure shows the real part of the wave vector as a function of free space wavelength for surface plasmon polaritons excited on single-crystal gold. The red symbols are results of fitting a LDOS model to the measurements. The solid gray line is the calculated dispersion expected from optical constants. The inset shows the measured CL intensity (red symbols) as a function of distance for a wavelength of $750 \mathrm{~nm}$. The solid blue line is calculated from the LDOS model of Eq. (2) with $R$ adjusted to fit the experimental data.

$$
\rho=\frac{\omega^{2}}{3 \pi^{2} c^{3}}+\frac{1}{2 \pi^{2} \omega} \operatorname{Im}\left\{E_{\perp}^{\mathrm{ind}} / D\right\},
$$

where the first term on the right-hand side is the vacuum LDOS. The normal-induced field $E_{\perp}^{\text {ind }}$ has a component due to the interaction of the dipole with the infinite planar surface and a distance-dependent contribution arising from reflection of SPPs at the grating,

$$
E_{\perp}^{\text {ind }}=E_{\perp}^{0}\left[1+\frac{1}{\sqrt{2 k_{\mathrm{SPP}} d}} \mathrm{R}^{2 i k_{\mathrm{SPP}} d}\right],
$$

where $k_{\mathrm{SPP}}$ is the momentum of the SPP and $R$ is the reflection coefficient of the grating. Equation (3) predicts oscillations with distance $d$ of period equal to half the SPP wavelength, in good agreement with our measurements.

We have calculated the local density of states from Eq. (2) under the approximation of Eq. (3) and adjusted $R$ to fit the data in Fig. 2. The inset of Fig. 4 shows the experimental CL intensity as a function of position from Fig. 2(a) for a wave- length of $750 \mathrm{~nm}$ together with the calculated relative LDOS from our model. The used fit parameters were the reflectivity of the grating and the SPP wave vector. Good agreement between model and measurements is achieved without any convolution with a spatial resolution, proving the high resolution of the CL imaging technique. The main panel of Fig. 4 shows the real part of the SPP wave vector extracted from these fits to the measurements for each wavelength. The experimentally determined SPP wave vectors agree well with the calculated dispersion using the dielectric function obtained from spectroscopic ellipsometry measurements of single-crystal gold. It should be noted that the present experiment probes the radiative part of (and not the absolute) LDOS as (i) SPPs exhibit losses, in particular, those incident to the grating at glancing incidence and (ii) in the present geometry only SPPs in one two-dimensional half space (i.e., those launched toward the grating) are collected and interfere with the full TR contribution.

In conclusion, we have shown that spatially resolved cathodoluminescence spectroscopy on single-crystalline gold shows oscillations in CL emission with distance from a grating. These oscillations are ascribed to the interference between outcoupled SPPs and transition radiation. This interference holds great potential for exploring absolute phase changes during SPP scattering in nanostructured surfaces and provides a direct measure for the photonic local density of states associated to SPPs. Due to the nanoscale resolution of the exciting electron beam, cathodoluminescence yields information on basic plasmon properties that is not accessible to any other technique. The current measurement of the photonic LDOS with unprecedented resolution is of fundamental interest in photonics, as the LDOS controls the efficiency of several useful phenomena involving light absorption and emission.

This work is part of the research program of FOM, which is financially supported by NWO. This work is also funded by NANONED. Work at Caltech is financially supported by the AFOSR under MURI with Grant No. FA9550-04-1-0434. F.J.G.A. acknowledges support from the Spanish MEC (Contract No. MAT2007-66050).
${ }^{1}$ H. Raether, Surface Plasmons on Smooth and Rough Surfaces and on Gratings (Springer, New York, 1988).

${ }^{2}$ M. I. Stockman, Phys. Rev. Lett. 93, 137404 (2004).

${ }^{3}$ H. J. Lezec, J. A. Dionne, and H. A. Atwater, Science 316, 430 (2007).

${ }^{4}$ N. Yamamoto, K. Araya, and F. J. García de Abajo, Phys. Rev. B 64, 205419 (2001).

${ }^{5}$ E. J. R. Vesseur, R. de Waele, M. Kuttge, and A. Polman, Nano Lett. 7, 2843 (2007).

${ }^{6}$ F. J. García de Abajo and M. Kociak, Phys. Rev. Lett. 100, 106804 (2008).

${ }^{7}$ J. T. van Wijngaarden, E. Verhagen, A. Polman, C. E. Ross, H. J. Lezec, and H. A. Atwater, Appl. Phys. Lett. 88, 221111 (2006).

${ }^{8}$ M. V. Bashevoy, F. Jonsson, A. V. Krasavin, N. I. Zheludev, Y. Chen, and M. I. Stockman, Nano Lett. 6, 1113 (2006).

${ }^{9}$ P. Goldsmith and J. V. Jelley, Philos. Mag. 4, 836 (1959).
${ }^{10}$ N. Yamamoto, H. Sugiyama, and A. Toda, Proc. R. Soc. London, Ser. A 452, 2279 (1996).

${ }^{11}$ G. W. Ford and W. H. Weber, Phys. Rep. 113, 195 (1984).

${ }^{12}$ F. J. García de Abajo and A. Howie, Phys. Rev. B 65, 115418 (2002).

${ }^{13}$ F. J. García de Abajo, Rev. Mod. Phys. 79, 1267 (2007).

${ }^{14}$ F. J. García de Abajo (to be published).

${ }^{15}$ L. D. Landau, E. M. Lifshitz, and L. P. Pitaevskii, Electrodynamics of Continuous Media (Pergamon, New York, 1984).

${ }^{16}$ D. Pacifici, H. J. Lezec, H. A. Atwater, and J. Weiner, Phys. Rev. B 77, 115411 (2008).

${ }^{17}$ K. H. Drexhage, J. Lumin. 1-2, 693 (1970).

${ }^{18}$ D. P. Fussell, R. C. McPhedran, and C. Martijn de Sterke, Phys. Rev. E 70, 066608 (2004).

${ }^{19}$ L. A. Blanco and F. J. García de Abajo, Phys. Rev. B 69, 205414 (2004). 